\title{
EDITORIAL
}

\section{The application of good clinical practice in nutrition research}

\author{
European Journal of Clinical Nutrition (2012) 66, 1280-1281; \\ doi:10.1038/ejen.2012.132
}

High-quality human studies are indispensable to obtain credible scientific evidence for nutritional effects on the structure and functioning of the human body in health and disease. Although nutrition studies can have specific characteristics in terms of populations, outcomes, designs, methodologies and interventions, ${ }^{1}$ it is clear that human nutrition research should follow the established basic scientific and operational principles for highquality design and execution of human studies. These aspects, together with those related to subject protection, form the basis of the ICH Good Clinical Practice (GCP) guidelines. ${ }^{2}$ Consequently, the GCP principles are relevant and important for human nutrition research. In fact, nearly all articles of the GCP guidelines can be applied to nutrition studies. However, some GCP aspects need a certain level of adaptation to be practically incorporated into nutrition trials. These are discussed in the current paper.

First, GCP contains certain definitions and articles that are related to the pharmaceutical origin of the ICH-GCP, which are incompatible to nutrition studies. First and foremost, GCP refers to clinical trials as testing an 'investigational product', meaning a 'pharmaceutical form of an active ingredient or placebo'. Interventions in nutrition trials rarely consist of well-defined single molecular entities, but instead often include complex ingredients, extracts or food products consisting of mixes of ingredients, or even full or partial dietary modifications. A broader interpretation of the term clinical trial that includes all human studies with an intervention, including nutrition and dietary modifications, that are aimed to gain medical, physiological or biological knowledge, would facilitate the adoption of GCP guidelines in nutrition and other relevant research. Consumer testing of non-experimental product acceptance, taste or liking would be out of scope under this definition.

Specific pharmaceutical or medical terminology and approaches can also be found in a limited number of specific GCP articles. For example, an 'Adverse Drug Reaction' is obviously not an appropriate term for an adverse event that is (possibly) related to a nutrition intervention. However, this terminology is not an obstacle to adopt the underlying concept in nutrition trials. More problematic is the section on the information that is to be provided in the Investigator Brochure (IB). This section calls for detailed information on the investigational product characteristics (physical, chemical and pharmacological properties), and in-depth overviews of pre-clinical and human findings on pharmacokinetics, safety and efficacy. Nutrition interventions cannot always be precisely characterized in terms of molecular composition, kinetics and specific experimental safety data, particularly when it involves common whole foods, ingredients or diets. Nutrition trials also are not necessarily preceded by pre-clinical work; many foods (food-substances) have a history of safe use and the study rationale may arise from, for example, epidemiological findings. The specific GCP requirements for the IB are therefore often not compatible with nutrition clinical trials. A more liberal format, maintaining the GCP IB principle that all relevant information on risks and safety precautions needs to be available to the investigator, should be applied for nutrition trials.

A more general barrier for GCP in nutrition research is that, in today's practice, GCP compliance typically involves an elaborate system of documentation, training, monitoring, data management and quality audits, which is highly costly, laborious and time consuming. There is considerable resistance from nutrition researchers to adhere to such 'pharmaceutical' GCP procedures, which are often considered unnecessarily cumbersome for the purpose of their studies. Interestingly, recent initiatives have been launched to decrease unnecessary complexity in pharmaceutical clinical trial procedures by adopting risk-based approaches. ${ }^{3-5}$ Quality and safety control measures should be proportionate to the study-specific risks. Nutrition trials often, but not necessarily always, pose lower risks in terms of safety of the interventions (when dealing with established foods and diets), vulnerability of the study populations (when investigating healthy adult volunteers) and type of measurements. An adequate level of subject protection and data integrity could be maintained by less stringent or simplified monitoring ${ }^{3}$ and targeted adverse event recording. ${ }^{5,6}$ An appropriate risk assessment should be conducted by the sponsor and investigator, and should include the safety risks and the risks of obtaining invalid study results, as well as the scientific, societal and business implications of unreliable findings. The nature and level of the procedures to limit identified risks should be clearly specified in the protocol for review and approval by the Institutional Review Board/ Independent Ethics Committee (IRB/IEC). Future guidance documents to define standardized risk assessment criteria and associated actions would benefit the sponsor, investigator as well as IRB/IEC, and ultimately the volunteers. Furthermore, the IRB/IECs may consider incorporating permanent or ad-hoc nutritional experts to aid the evaluation of the particulars of nutrition study protocols.

GCP is equally important for nutrition trials as it is for pharmaceutical trials, and is-with the aforementioned remarksequally applicable to nutrition trials. GCP offers the flexibility to adopt a proportionate approach that will not hinder innovation and scientific knowledge gathering, but will stimulate high quality and safe nutrition research. It is up to investigators and sponsors in the nutrition area to use this flexibility to make the implementation of GCP fit to the specific requirements and risks involved in their research. This should be done without an excessive administrative burden. Such interpretation should be positively regarded and accepted by the relevant ethics committees and authorities.

\section{CONFLICT OF INTEREST}

The authors declare no conflict of interest.

\section{ACKNOWLEDGEMENTS}

This publication was commissioned by the Functional Foods Task Force of the European branch of the International Life Sciences Institute (ILSI Europe). Industry members of this task force are Abbott Nutrition, Barilla G.\& R. Fratelli, BASF, Bionov Biosearch Life, Campbell Soup Company, Cargill, Chiquita Brands International, CocaCola Europe, Danone, DOW Europe, DSM, DuPont de Nemours, Kellogg Europe, Kraft Foods Europe, International Nutrition Company, Institut Mérieux, Mars, McNeil Nutritionals, Naturex, Nestlé, Newtricious, PepsiCo International, Pfizer Consumer Healthcare, Red Bull, Royal FrieslandCampina, Rudolf Wild, Schwabegroup, Soremartec Italia - FERRERO Group, Südzucker/BENEO Group, Tate \& Lyle Ingredients, TereosSyral, Unilever, Yakult Europe. This publication was coordinated by Dr Alessandro Chiodini, Scientific Project Manager at ILSI Europe. 


\section{DISCLAIMER}

The opinions expressed herein and the conclusions of this publication are those of the authors and do not necessarily represent the views of ILSI Europe nor those of its member companies.

JAJ Schmitt ${ }^{1,2}, \mathrm{H}$ Bouzamondo ${ }^{3}, \mathrm{~F}$ Brighenti ${ }^{4}$, AK Kies $^{5}$, I Macdonald ${ }^{6}$, AFH Pfeiffer ${ }^{7,8}$ and A Chiodini ${ }^{9}$ ${ }^{1}$ Nestlé, Clinical Development Unit, Lausanne, Switzerland; ${ }^{2}$ Centre for Human Psychopharmacology, Swinburne University, Melbourne, Victoria, Australia;

${ }^{3}$ Unilever, Vlaardingen, The Netherlands; ${ }^{4}$ University of Parma, Department of Food Science, Parma, Italy; ${ }^{5}$ DSM, Biotechnology Center, Delft, The Netherlands; ${ }^{6}$ University of Nottingham, Medical School, Nottingham, UK; ${ }^{7}$ German Institute of Human Nutrition-Potsdam, Brandenburg, Germany; ${ }^{8}$ Charité University Medicine, Department of Clinical Nutrition, Berlin, Germany and ${ }^{9}$ ILSI Europe, Brussels, Belgium E-mail: publications@ilsieurope.be

\section{REFERENCES}

1 Welch RW, Antoine JM, Berta JL, Bub A, de Vries J, Guraner F et al. Guidelines for the design, conduct and reporting of human interventions studies to evaluate the health benefits of foods. Br J Nutr 2011; 106: S4-S15.

2 ICH. Guideline for Good Clinical Practice E6(R1) 1996. http://www.ich.org/fileadmin/ Public_Web_Site/ICH_Products/Guidelines/Efficacy/E6_R1/Step4/E6_R1_Guideline. pdf.

3 U.S. Department of Health and Human Services Food and Drug Administration. Guidance for industry. Oversight of clinical investigations-a risk-based approach to monitoring 2011. www.fda.gov/downloads/Drugs/GuidanceComplianceRegulatorylnformation/Guidances/UCM269919.pdf.

4 European Medicines Agency. Reflection paper on risk based quality management in clinical trials 2011. www.ema.europa.eu/docs/en_GB/document_library/Scientific_guideline/2011/08/WC500110059.pdf.

5 Medicines and Healthcare Products Regulatory Agency. Risk-adapted approaches to the management of clinical trials of investigational medicinal products 2011. www.mhra.gov.uk/Howweregulate/Medicines/Inspectionandstandards/GoodClinical Practice/News/CON126145.

6 Yusuf S. Damage to important clinical trials by over-regulation. Clin Trials 2010; 7: 622-625. 